\title{
Acknowledgement to Reviewers of Nutrients in 2017
}

\author{
Nutrients Editorial Office \\ MDPI AG, St. Alban-Anlage 66, 4052 Basel, Switzerland \\ Published: 16 January 2018
}

Peer review is an essential part in the publication process, ensuring that Nutrients maintains high quality standards for its published papers. In 2017, a total of 1340 papers were published in the journal. Thanks to the cooperation of our reviewers, the median time to first decision was 21 days and the median time to publication was 53 days. The editors would like to express their sincere gratitude to the following reviewers for their time and dedication in 2017:

Aagaard, Niels Kristian

Aarabi, Mahmoud

Aaron, Jean E.

Abe, Sumiko

Abedelmalek, Salma

Abenavoli, Ludovico

Abi Nader, Patrick

Abkowitz, Janis L.

Aboud, Frances

Abraham, Bincy P.

Abraham, Christie

Abrahamsson, Thomas

Abt, Michael C.

Aceña, Álvaro

Acuña-castroviejo, Dario

Adamkova, Anna

Adegboye, Amanda

Adekanmbi, Victor T.

Adhami, Vaqar M.

Adriouch, Solia

Aerenhouts, Dirk

Afrin, Sadia

Aggarwal, Abhishek

Aggarwal, Bharat B.

Ágh, Tamás

Aghajafari, Fariba

Agil, Ahmad

Agostoni, Carlo

Aheto, Justice Moses K.

Ahluwalia, Amrita

Ahmet, Ismayil

Aires, Alfredo

Alaedini, Armin

Alaofe, Halimatou

Albonici, Loredana
Albrecht, Sandra S.

Aldawsari, Fahad

Al-Dujaili, Emad

Alexy, Ute

Al-Hamdani, Mohammed

Allegaert, Karel

Allen, Andrew

Almajano, María Pilar

Al-Nakkash, Layla

Alonso-Magdalena, Paloma

Alsharairi, Naser

Altamura, Sandro

Altieri, Barbara

Aluko, Rotimi

Álvarez, Carlos

Alwahsh, Salamah M.

Ambigapathy, Ganesh

Amedei, Amedeo

Amoaku, Winfried M.

Amodio, Piero

Anadón, Arturo

Ananieva, Elitsa

Anastasia, Vlasova

Andersen, Barbara Vad

Andersen, Catherine J.

Anderson, Cheryl

Anderson, Peter

Andreas, Nicholas

Aneskievich, Brian J.

Anestis, Dougkas

Angadi, Siddhartha

Angeles Martin, Maria

Angelino, Donato

Angelucci, Adriano

Annibale, Bruno 
Annunziata, Azzurra

Ansari, Rais

Anthias, Chloe

Anthimopoulos, Marios

Anthony, Tracy

Antoine-Jonville, Sophie

Antonakou, Angeliki

Antonio, Jose

Anvari, Sara

Aoi, Wataru

Appleton, Katherine

Araújo, João Ricardo

Arboleya, Silvia

Archer, Edward

Arcidiacono, Biagio

Arciero, Paul

Ardenghi, Diego

Ardévol, Anna

Arfuso, Frank

Ariño, Agustín

Ariyoshi, Nobuhiro

Arjmandi, Bahram

Armand, Martine

Armanini, Decio

Armstrong, Gavin R.

Arrick, Denise

Arteaga, Olatz

Arulselvan, Palasamy

Arús, Carles

Asahina, Kinji

Asano, Shinichi

Aschbrenner, Kelly

Ashcroft, Frances

Ashman, Amy

Aspalter, Rosa

Aspatwar, Ashok

Aterini, Stefano

Atkins, Janice L.

Atkinson, Stephanie

Attanasio, Chiara

Atteritano, Marco

Attuquayefio, Tuki

Auguet, Teresa

Augustin, Hanna

Augustin, Livia

Aune, Dagfinn

Auricchio, Salvatore

Austin, Gregory

Avery, Amanda

Avignone-Rossa, Claudio

Axelsson, John

Ayonrinde, Oyekoya
Ayton, Scott

Azarpazhooh, Elham

Azcárate-Peril, M. Andrea

Azcona, María Cristina

Azmi, Asfar S.

Azzout-Marniche, Dalila

Baar, Keith

Baba, Hideo

Bach, Christopher

Baecker, Natalie

Baenas, Nieves

Bagüés, Ana

Bahwere, Paluku

Bailey, Lynn

Bailey, Regan

Bains, Pushpinder

Baïz, Nour

Bajka, Balazs

Bakardjian, Hovagim

Baker, Julien

Bakillah, Ahmed

Bakker, Barbara

Baksh, Shairaz

Balantekin, Katherine

Balcerczyk, Aneta

Baldassarre, Maria Elisabetta

Baldofski, Sabrina

Ballmann, Christopher

Ballocca, Flavia

Bally, Lia

Bana, Jinan

Banach, Maciej

Banerjee, Aditi

Banerjee, Hirendra Nath

Banerjee, Kalpita

Bao, Yongping

Bapputty, Reena

Baragetti, Andrea

Baranovski, Tom

Barbaresko, Janett

Barbul, Adrian

Barchetta, Ilaria

Bärebring, Linnea

Barffour, Maxwell

Barker, Edward D.

Barker, Tyler

Barnard, Neal

Barrea, Luigi

Barreira, João C. M.

Barrero, Anna

Bartelt, Alexander

Barvencik, Florian 
Baskaran, Charumathi

Bassareo, Pier Paolo

Basso, Kari

Bates, Christopher

Baudry, Julia

Bauer, Judith

Baum, Jamie

Baur, Daniel A.

Baute, Vanessa

Bayat, Vafa

Bazzano, Alessandra

Beasley, Shea

Beberashvili, Ilia

Beck, Kathryn

Beckett, Emma

Bedard, Annabelle

Bednářová, Martina

Beeken, Rebecca

Beezhold, Bonnie

Belay, Brook

Bell, Colin

Bell, David

Bell, Jack J.

Bell, Kirstine

Bellisle, France

Bello, Nicholas T.

Belury, Martha

Benatar, Jocelyne

Bendik, Igor

Benedec, Daniela

Benn, Christine Stabell

Bentel, Jacqueline M.

Benzeroual, Kenza E.

Berens, Pamela D.

Bergdahl, Andreas

Berger, Mette M.

Bergheim, Ina

Berglund, Staffan

Bergström, Anna

Bering, Stine Brandt

Berkley, James A.

Bernard, Jonathan

Bernardo, Ana Paula

Bernasconi, Pia

Bernhard, Wolfgang

Berni Canani, Roberto

Bernlohr, David A.

Bernstein, Melissa

Berretta, Massimiliano

Berrington, Janet

Berry, Marla

Berry, Narelle
Bertolo, Robert

Bertram, Hanne Christine

Best, Karen

Beto, Judith A.

Bettaieb, Ahmed

Beverly, Levi J.

Bezirtzoglou, Eugenia

Bharadwaj, Shishira

Bhatia, Jatinder

Bhattacharjee, Sonali

Bhattacharya, Sayak

Bhavsar, Amit P.

Biank, Vincent F.

Bidel, Siamak

Bidwell, Amy J.

Bienertova-Vasku, Julie

Biesiekierski, Jessica

Bilal, Usama

Bingham, Richard

Birch, Laura

Bird, Amanda

Birrell, Mark

Bisanz, Jordan

Bishayee, Anupan

Bishop, Karen

Bittinger, Kyle

Bitto, Alessandra

Bix, Laura

Bjørke Monsen, Anne-Lise

Bjørndal, Bodil

Blachier, François

Black, Lucinda

Black, Maureen M.

Blais, Anne

Blando, Federica

Blaner, William S.

Blanton, Cynthia

Blesso, Christopher

Block, Galdys

Bo, Simona

Board, Mary

Boaz, Mona

Bodet, Charles

Boekema, Bouke

Bogl, Leonie Helen

Bøgwald, Jarl

Bøhn, Siv K.

Boland, Michael

Bonaccio, Marialaura

Bonaccorsi, Guglielmo

Bonavina, Luigi

Bondonno, Catherine P. 
Bondonno, Nicola P.

Bondy, Stephen C.

Bongers, Kale

Bonilla Ocampo, Diego A.

Bonilla, Carolina

Bonomini, Francesca

Bonos, Eleftherios

Boquien, Clair-yves

Bora, Puran S.

Borbély, Yves

Borengasser, Sarah J.

Borer, Katarina T.

Bosch Ruiz, Marc

Bosch, Christine

Bosnjak, Berislav

Botelho, Goreti

Botham, Kathleen

Bottoms, Lindsay

Boucher, Barbara J.

Boushey, Carol

Bowen, Deborah J.

Bowling, April

Boyer, Constance

Boyle, Robert J.

Bradbury, Kathryn E.

Bradshaw, Patrick

Brandhagen, Martin

Brandt, Kirsten

Brantsæter, Anne Lise

Branum, Amy M.

Braun, Kim V. E.

Bravenboer, Nathalie

Bravo, Laura

Bray, George

Brecht, Marcus

Breen, Cathy

Breuillard, Charlotte

Brewer, George J.

Brewster, Lizzy M.

Briassoulis, George

Briend, André

Briggs, Marc A.

Brighenti, Susanna

Bril, Fernando

Brinkley, Tina

Briskey, David

Broderick, Tom L.

Brookes, Denise S. K.

Broom, Iain

Brosnihan, K. Bridget

Broszczak, Daniel

Brottveit, Margit
Brough, Louise

Brown, Katie

Brown, Kyle E.

Brown, LaVerne L.

Brown, Leanne

Brown, Paula

Brown, Rachel

Brownell, Elizabeth

Brownlee, Iain A.

Bruce-Keller, Annadora J.

Bruckbauer, Antje

Bruneau, Michael Jr.

Bruns, Danielle R.

Bruyndonckx, Luc

Buccigrossi, Vittoria

Bucher, Tamara

Buckland, Nicola

Buddington, Randal

Buechler, Christa

Bügel, Susanne

Bugliani, Marco

Bührer, Christoph

Bukong, Terence N.

Bunn, Diane

Burden, Richard

Burgess, John R.

Burghardt, Kyle Jon

Burkhart, Sarah

Burkhead, Jason

Burns, David

Burri, Betty J.

Burrows, Tracy

Burtey, Stéphane

Burton, E. Thomaseo

Burton, Jeremy P.

Buscail, Camille

Butel, Marie-José

Butler, Tom

Butterworth, Peter J.

Buyken, Anette E.

Byberg, Liisa

Bybrant, Mara Cerqueiro

Byrne, Rebecca

Cabanillas, Beatriz

Cabezas, Manuel Castro

Cadario, Francesco

Cadeau, Claire

Caffarelli, Carlo

Cai, Demin

Cai, Hui

Cai, $\mathrm{Lu}$

Cai, Shirong 
Cairrão, Elisa

Calani, Luca

Calder, Philip

Caldovic, Ljubica

Calkins, Kara L.

Camire, Mary Ellen

Campbell, Matthew D

Camps, Jordi

Canella, Daniela Silva

Canfora, Emanuel E.

Cannell, John J.

Capaldo, Brunella

Capel, Frédéric

Capewell, Simon

Capitanio, Nazzareno

Caporaso, Nicola

Capuani, Silvia

Cardenas, Andres

Cardillo, Carmine

Cardoso, Luís

Cardoso, Susana

Carlberg, Carsten

Carlsen, Monica H.

Carnevale, Daniela

Carocho, Márcio

Caroli, Anna.

Caron, Joan McIntyre

Carpene, Christian

Carr, William H

Carradori, Simone

Carrillo-Sepulveda, Maria Alicia

Casanovas, Carmen

Cascone, Sara

Casperson, Shanon L.

Cassetta, Luca

Castell, Lindy

Castellazzi, Anna Maria

Castello, Adela

Castrogiovanni, Paola

Catalano, Antonino

Catassi, Carlo

Cauli, Omar

Ceccarelli, Sara

Ceolotto, Giulio

César, Carina

Cesareo, Roberto

Chachay, Veronique S.

Chae, Heeyoung

Chambers, Edgar IV

Chan, Catherine B.

Chan, Julie

Chan, Maria
Chand, Sourabh

Chandra-Hioe, Maria Veronica

Chang, Chen-Kang

Chang, Chuang-Rung

Chang, Eugene B.

Chang, Fang-Rong

Chang, Hsin-I

Chang, Jer-Ming

Chang, Jung-Su

Chang, Sue-Joan

Chao, Ariana M.

Chapple, Sarah

Chassard, Christophe

Chaung, Hso-Chi

Chauveau, Philippe

Chavarro, Jorge

Chaves-Lopez, Clemencia

Chen, Eunice

Chen, Guibing

Chen, Guoxun

Chen, Jih-Jung

Chen, Ling-Wei

Chen, Qi

Chen, Ruei-ming

Chen, Shiu-Nan

Chen, Shuai

Chen, Szu-Chia

Cheng, I-Shiung

Cheng, Juei-tang

Cheng, Ken

Cheng, Kuang-Hung

Cheng, Nai-Chen

CHENG, Wen-Hsing

Cheng, Xianwu

Chengwen, Sun

Cherasse, Yoan

Cherbuy, Claire

Cherniack, Evan Paul

Cheungpasitporn, Wisit

Chiang, Chien-Hsieh

Chiang, Meng-Tsan

Chiarioni, Giuseppe

Chiavaroli, Laura

Chiechio, Santina

Chien, Yi-Wen

Chieppa, Marcello

Chilibeck, Phil

Chiou, Wen-Fei

Chirumbolo, Salvatore

Chitchumroonchokchai, Chureeporn

Chiurchiù, Valerio

Chmurzyńska, Agata 
Cho, Clare

Cho, Jinmyoung

Cho, Ssang-Goo

Chobot, Vladimir

Choi, Myung-Sook

Chong, Mary Foong-Fong

Choudhury, Malay

Christen, William G.

Christides, Tatiana

Christmann, Viola

Christodoulou, Maria-Ioanna

Christophersen, Claus

Chruszcz, Maksymilian

Chu, Anna

Chu, Shidong

Chua, Wei-Hang

Chun, Ock K.

Chun, Rene

Chung, Louisa Ming Yan

Ciacci, Carolina

Cibulskis, Catherine

Ciccocioppo, Rachele

Cicero, Arrigo

Ciclitira, Paul

Cifelli, Christopher J.

Cifuentes, Liliana

Cione, Erika

Clark, William

Clarke, Neil

Clayton, David

Clemens, Roger

Clemens, Zsófia

Clemente, Maria Grazia

Clifton-Bligh, Phillip B

Coates, Penelope

Cochran, Blake

Cohen, Ronald

Collado, Pilar Sánchez

Colleran, Heather L.

Collings, Paul

Colussi, GianLuca

Coman, Maria Magdalena

Combet, Emilie

Comelli, Elena

Conceição, Eva

Concors, Seth Jason

Conrad, Zach

Considine, Robert

Conti, Pio

Cooke, Matt

Coppedè, Fabio

Cordaro, Marika
Cordero, Paul

Corish, Clare

Corley, Michelle M.

Cornelis, Marilyn C.

Corniola, Rikki

Cornish, M. Lynn

Cornish, Stephen

Corpe, Christopher

Cortés-Castell, Ernesto

Corthay, Alexandre

Costantini, Susan

Costa-Rodrigues, João

Costello, Rebecca Bortz

Coughlan, Melinda T.

Courtney-Martin, Glenda

Covasa, Mihai

Cravero, Maria Carla

Crespo, Irene

Croft, Kevin

Crossland, Nicola

Cubero, Francisco Javier

Cullers, Andrea

Cullerton, Katherine

Cumming, Kristoffer T.

Cunha, Rodrigo

Cunningham-Rundles, Susanna

Cutuli, Debora

Czikora, Istvan

D'Addario, Claudio

Da Paz, Vanessa Ribeiro Figliuolo

Dahech, Imen

Dalbeni, Andrea

Dalbo, Vincent

D'Alessio, Patrizia A.

Dalla Rosa, Marco

Dall' Acqua, Stefano

Dalmas, Elise

Dalsgaard, Trine Kastrup

Dalton, Caroline

Danesi, Francesca

Dankel, Simon N.

D'archivio, Massimo

Darling, Andrea L.

darmon, nicole

Darvin, Maxim

Das, Amitava

Das, Undurti

Dashti, Hassan

D'Auria, Enza

D'Auria, Maria Valeria

Davidson, Rose

Davila, Anne-Marie 
Davinelli, Sergio

Davison, Karen

Davy, Brenda

Davy, Kevin P.

Dawood, Mahmoud A. O.

De Borst, Martin H.

De Cock, Nathalie

De Geest, Bart

De Gruijl, Frank

De Kreutzenberg, Saula

De La Cruz, José Pedro

De La Monte, Suzanne

De La Parra, Columba

De Las Heras, Javier

De Leo, Vincenzo

De Lorgeril, Michel

De Magistris, Laura

De Magistris, Tiziana

De Mestral Vargas, Carlos

De Pascual-Teresa, Sonia

De Smet, Stefaan

De Sotomayor, Maria Alvarez

De Steur, Hans

De Vito, Elisabetta

De Vlieger, Nienke

De Vries, Jeanne

De Wild, Victoire W. T.

Dean, Lisa

De-Bandt, Jean-Pascal

DeBusk, Ruth

Deeth, Hilton C.

Dekker, Marloes

Del Bo, Cristian

Del Giudice, Rita

Del Rio, Daniele

DeLany, James

Delarue, Jacques

Deli, Chariklia K.

Delisle, Hélène

Della Torre, Sara

Dellavalle, Diane M.

Delmastro-Greenwood, Meghan

Delvin, Edgard E.

Den Hartigh, Laura J.

Denham, Bryan E.

Deo, Permal

Depner, Christopher Michael

Depoortere, Inge

Derave, Wim

Derraik, José G. B.

Desai, Mahesh S.

Deschasaux, Mélanie
Després, Jean-Pierre

Desselberger, Ulrich

Detzel, Patrick

Deutsch, Jonathan

Deutz, Nicolaas E. P.

Dev, Dipti

Devesa, Vicenta

Devlin, Angela

Dey, Moul

Di Biase, Stefano

Di Daniele, Nicola

Di Felice, Gabriella

Di Gioia, Diana

Di Iorio, Biagio Raffaele

Di Nardo, Giovanni

Di Palma, Michael

Dickerson, Roland N.

Dickinson, Kacie

Dilger, Ryan

Dillon, E. Lichar

Dinesen, Pia Thisted

Dinh, Dzung H.

DiNicolantonio, James J.

Dinu, Monica

Dioum, Elhadji M.

Djuric, Zora

Dobrosielski, Devon A.

Doig, Gordon S.

Dolinsky, Vernon W.

Dominguez, Ligia J.

Dominguez-Perles, Raul

Donadio, Carlo

Donne, Bernard

Donno, Dario

Donofry, Shannon D.

Donovan, Sharon

Doshi, Simit

Dossus, Laure

Dötsch-Klerk, Mariska

Doyle, Todd

Dragsted, Lars Ove

Dreher, Mark

Drevon, Christian A

Drew, Janice E.

Drobnic, Franchek

Drossard, Claudia

Duckworth, Lauren C.

Dufour, Darna L.

Dullaart, Robin P. F.

Dumler, Francis

Dumont, Patrick

Dunford, Elizabeth 
Duntas, Leonidas

Durazo-Arvizú, Ramón Angel

Durazzo, Alessandra

During, Alexandrine

Duryee, Michael J.

Dusso, Adriana

Duwaerts, Caroline

Earnest, Conrad P

Eastman, Creswell

Eaton, Philip

Ebeling, Peter

Eduardo-Figueira, Maria

Edurne, Simón Magro

Efird, Jimmy

Egger, Garry

Ehret, Georg B.

Einöther, Suzanne J. L.

Ekuni, Daisuke

Elaine Hardman, W.

Eldridge, Alison L.

Eleftheriadis, Theodoros

Elisabet, Forsum

Ellervik, Christina

Elli, Luca

Elli, Marina

Elliott, Michael B.

El-Salhy, Magdy

El-Seedi, Hesham R.

Elsheikha, Hany M.

el-Sohemy, Ahmed

Empl, Michael

Engle-Stone, Reina

Ennis, Julie Mason

Enomoto, Hirayuki

Erdman, John

Eri, Raj

Eri, Rajaraman

Ericsson, Madelene

Eriksson, Johan G.

Ershow, Abby G.

Erzinger, Melanie

Escolà-Gil, Joan Carles

Esposito, Ciro

Esworthy, Steve

Euston, Stephen

Evans, Christian C.

Evans, E Whitney

Evans, Gethin

Evans, Joseph L.

Everaert, Nadia

Evin, Geneviève

Eyles, Darryl W.
Ezedine, Bouhlel

Ezekiel, Uthayashanker

Fabbricatore, Mariantonietta

Fabiani, Roberto

Factor-Litvak, Pam

Fagerstedt, Kurt

Fahey, George

Fairchild, Timothy John

Fairweather, Stephen

Fairweather-Tait, Susan J.

Fang, Jim

Fanizzi, Francesco P.

Fargion, Silvia

Farhat, Grace

Farina, Emily K.

Farr, Susan A.

Farran-Codina, Andreu

Farrar, Mark

Fayet-Moore, Flavia

Feas, Xesus

Fedosov, Sergey

Feinman, Richard D.

Felder, Robin A.

Feldman, David

Felt, Barbara

Feng, Yibin

Fenton, Tanis

Feo, Francesco

Feresin, Rafaela G.

Ferlazzo, Nadia

Fernandes, Eduarda

Fernandes, Maria Fernanda

Fernandez, Maria Luz

Fernandez-Aranda, Fernando

Fernández-Sánchez, María Luisa

Ferramosca, Alessandra

Ferrario, Chiara

Ferraris, Ronaldo P.

Ferraro, Pietro Manuel

Ferreira, Isabel

Ferreira, Rui M.

Ferrell, Jessica M.

Ferrer-García, Marta

Ferrie, Suzie

Fetissov, Sergueï O.

Fetterman, James W.

Fiaccadori, Enrico

Field, Catherine

Fields, David

Fildes, Alison

Filler, Guido

Fine, Eugene J. 
Fine, James Burke

Fineschi, Vittorio

Finley, John

Fiocchi, Alessandro

Fiocco, Daniela

Firrman, Jenni Ann

Flack, Kyle D.

Flechtner-Mors, Marion

Fleenor, Bradley S.

Fleig, Lena

Flexeder, Claudia

Floch, Martin H.

Flueck, Joelle L.

Flynn, Charles R.

Foegeding, E. Allen

Fogelholm, Mikael

Fölster-Holst, Regina

Fontana, Luis

Foong, Rachel E.

Foote, Janet A.

Forbes, Scott. C.

Forman, Michele

Forrest, Lauren

Forsmark, Chris E.

Forsyth, Adrienne

Fortenberry, Megan

Foster, Charles B.

Foster, Meika

Fouladkhah, Aliyar

Fourtounas, Costas

Fox, Simon

Fragkos, Konstantinos

Fragopoulou, Elizabeth

Frame, Leigh A.

Franco, Carlos

Franklin, Peter

Franko, Andras

Frazer, David

Frazzi, Raffaele

Freeman, Linnea R.

Freije, José M. P.

Friel, James

Friesen, Carol

$\mathrm{Fu}$, Tzu-Fun

Fuge, Ron

Fujihara, Hisako

Fujimori, Ko

Fujita, Mikako

Fukata, Masayuki

Fukuda, Mitsuru

Fukuda, Yoshiharu

Fukui, Michiaki
Fulgoni, Victor L. III

Fullerton, Morgan

Funato, Hiromasa

Fung, Ellen B.

Fürnsinn, Clemens

Fusaro, Maria

Gachon, Frederic

Gadgil, Meghana D.

Gahche, Jaime

Galanis, Alex

Galeazzi, Gian Maria

Galli, Francesco

Galvano, Fabio

Gálvez, Julio

Gan, Ren-You

Ganapathy, Vadivel

Gander, Jennifer

Gandy, Joan

Gangloff, Anne

Ganji, Vijay

Gao, Jie

Garcia De La Hera, Manoli

Garcia, Ada

García, Marta Mesías

Garcia-Bailo, Bibiana

García-Martínez, Eva

García-Parrilla, Maria.C.

García-Sánchez, Asunción

Gard, Paul

Garden, Frances

Garden-Robinson, Julie

Garg, Manohar

Garnacho-Castaño, Manuel Vicente

Garza, Kimberly

Gasparrini, Massimiliano

Gassler, Nikolaus

Gaudette, Nicole J.

Gaudichon, Claire

Gaultier, Alban

Gay-Quéheillard, Jerome

Ge, Dongxia

Ge, Xiaodong

Gea, Alfredo

Gebhardt, Rolf

Geisler, Corinna

Genoni, Angela

Gentili, Sheridan

Georgiev, Vasil

Gérard, Philippe

Gerards, Sanne

Gerber, Leonard E.

Gerber, Mariette 
Ghassabian, Akhgar

Ghatak, Arindam

Giaginis, Constantinos

Giamarellos-Bourboulis, Evangelos J.

Giampieri, Francesca

Gianfrani, Carmen

Gianini, Loren M.

Giannenas, Ilias

Gianni, Maria Lorella

Giapros, Vasileios

Giavaresi, Gianluca

Gibney, Eileen

Gibson, Alice. A.

Gibson, Peter

Gibson, Simone

Giel, Katrin

Giera, Martin

Gifford, Janelle A.

Giles, Grace E.

Gill, Tiffany

Gillevet, Patrick

Giordano, Mauro

Giudetti, Anna

Gkimpas, Georgios

Gkolfakis, Paraskevas

Głąbska, Dominika

Glade, Michael J.

Gladine, Cecile

Glahn, Ray

Glaister, Mark

Glenn, Melissa J.

Glenn, Thomas C.

Go, Gwang-Woong

Godfrey, Henry P.

Godos, Justyna

Goetz, Margarethe

Goglia, Fernando

Goicoa, Tomás

Goldbohm, R. Alexandra

Gold-Smith, Fuchsia

Goletzke, Janina

Golley, Rebecca

Golovko, Mikhail

Gomez, Nidia

Gómez-Ambrosi, Javier

Gómez-Zorita, Saioa

Gomollon, Fernando

Gonçalves, Guilherme

Gonzalez, Adam M.

Gonzalez, Javier T.

González, Sonia

Gonzalez-Barcala, Francisco Javier
Gonzalez-Casanova, Ines

González-Reimers, Emilio

Goodman, Richard

Gorham, Edward

Gorman, Shelley

Gower, Barbara

Goya, Luis

Grace-Farfaglia, Patricia

Graff, Rebecca E.

Graham, Daniel J.

Gramazio, Pietro

Gramlich, Leah

Granados-Principal, Sergio

Grant, William

Grasa, Laura

Grases, Felix

Grasselli, Elena

Grech, Amanda

Green, Peter

Greene, Michael W.

Greenhaff, Paul

Greenhalgh, David

Greenway, Frank L.

Greenwood, Darren C.

Greenwood, Michael T.

Gregori, Dario

Gregory, Jesse

Greibe, Eva

Gresham, Ellie

Grether-Beck, Susanne

Grider, Arthur

Grieger, Jessica A.

Griffin, Ian

Griffith, May

Grilli, Ester

Grim, Clarence E.

Grimes, Carley

Grisk, Olaf

Grootaert, Charlotte

Grosso, Clara

Grosso, Giuseppe

Grundy, Myriam M. L.

Grungreiff, Kurt

Grünler, Jacob

$\mathrm{Gu}$, Feng

Guarino, Alfredo

Guarino, Maria

Guasch-Ferre, Marta

Gudbrandsen, Oddrun A.

Gudey, Shyam Kumar

Gueant, Jean-Louis

Gueimonde, Miguel 
Guenther, Patricia M.

Guerre, Philippe

Guglielmetti, Simone

Gugliucci, Alejandro

Guidetti, Margherita

Guido, Luis F.

Guiné, Raquel P. F.

Gunst, Jan

Guo, Weimin

Gupta, Priya M.

Gutiérrez-Juárez, Roger

Gutierrez-Merino, Carlos

Gutiérrez-Orozco, Fabiola

Haapala, Eero A.

Haarmann-Stemmann, Thomas

Haase, Hajo

Haboubi, Nadim

Haczeyni, Fahrettin

Hadjikakou, Michalis

Hadjisavvas, Andreas

Hagenlocher, Yvonne

Hagman, Emilia

Hajduch, Eric

Haldar, Sumanto

Hall, Marcia.

Hall, Ulrika Andersson

Halloran, Bernard P.

Hamaguchi, Masahide

Hambidge, Michael

Hamden, Khaled

Hamdy, Osama

Hamlin, Robert

Hamner, Heather

Hampel, Daniela

Han, Jaehong

Han, Seung Jin

Han, Sung Nim

Han, Zhiyong

Hand, Timothy

Haneklaus, Moritz

Hannibal, Luciana

Hanson, Corrine

Hanson, Karla L.

Hao, Lei

Hara, Hiroshi

Hardman, Roy J.

Hardy, Melinda

Haring, Bernhard

Harnack, Lisa

Haron, Mona

Harris, Margaret

Harris, William
Harrison, Fiona

Hart, Andrew R.

Hart, Kathryn

Hart, Prue H.

Härtel, Christoph

Hartmann, Christina

Hartmann, Phillipp

Haschke, Ferdinand

Hasegawa, Yukihiro

Hashem, Kawther M.

Hashimoto, Naoto

Haskell-Ramsay, Crystal

Hassan, Iman

Hassan-Smith, Zaki

Hassouna, Rim

Hatsu, Irene

Hatta, Hideo

Hawinkels, Lukas

Hay, Phillipa

Hayes, Alan

Hayes, Kenneth C.

Haytowitz, David B.

Hearst, Mary O.

Heathers, James A. J.

Hebbard, Lionel

Heberden, Christine

Hecht, Jaqueline

Hector, Amy J.

Hedrick, Valisa

Hefferon, Kathleen Laura

Heianza, Yoriko

Heinen, Mirjam

Heinonen, Ilkka

Heiss, Christian

Hekmatdoost, Azita

Heller, Martin C.

Hellmann, Jason L.

Hellwig, Michael

Helms, Eric R.

Hemilä, Harri

Hendrich, Suzanne

Hendrie, Gilly

Heneberg, Petr

Hennessy, Áine

Herman, Mark

Hernandez, Maria

Hernandez, Teri

Hernández-Ledesma, Blanca

Herrera, Emilio

Herrick, Kirsten

Hess, Sonja

Hewlings, Susan J. 
Hida, Azumi

Hidaka, Brandon $\mathrm{H}$.

Hider, Robert

Hietikko, Elina

Higashida, Kazuhiko

Higgins, Matt

Hildebrandt, Herbert

Hiles, Sarah

Hill Gallant, Kathleen

Hill, James

Himotoe, Takashi

Hioki, Hirofumi

Hirko, Kelly

Ho, Chi-Tang

Ho, Su-Chen

Hocher, Berthold

Hodge, Allison

Hodgson, Jonathan

Hoerr, Robert

Hoerster, Katherine D.

Hoffer, Leonard John

Hoffman, Richard

Hokama, Tomiko

Holick, Michael

Holliday, Adrian

Holliday, Elizabeth G.

Hollis, Bruce

Holmes, Geoffrey

Holst, Jens Juul

Holst, Mette

Holt, Roberta R.

Holt, Tim A.

Holvik, Kristin

Hong, Mee Young

Hönow, Ruth

Hoogeveen, Ellen K.

Hook, Ingrid

Hopcraft, Matthew S.

Horax, Ronny

Horrigan, Louise A.

Hosick, Peter

Hosohata, Keiko

Hossein-Nezhad, Arash

Hotz, Christine

Hou, Yu-Chih

Houston, Mark C.

Howarth, Gordon

Howe, Anna

Hruz, Paul W.

Hsiang, Chien-Yun

Hsiao, George

Hsieh, Shu-Chen
Hsieh, Tusty-Jiuan

Hsu, Shu-hao

$\mathrm{Hu}$, Tian

Huab, Mark

Huang, Chi-Chang

Huang, Shih-Yi

Huang, Zhi

Huc, Laurence

Huggins, Catherine E.

Hughes, Catherine

Hughes, David

Hughson, Michael D.

Hull, Mark A.

Hulthén, Lena

Hummel, Sandra

Huq, Laila

Hur, Sun-Jin

Huss, Fredrik

Huston, Robert K.

Hutchinson, Jayne

Huysentruyt, Koen

Hypponen, Elina

Ide, Kazuki

Ideraabdullah, Folami Y.

Idrus, Nirelia

Iizuka, Katsumi

Ijiri, Daichi

IJzerman, Richard

Ikegaya, Naoki

Ilich-Ernst, Jasminka

Imai, Toshio

Imperatori, Claudio

Inagi, Reiko

Inestrosa, Nibaldo C.

Inman, Denise $\mathrm{M}$.

Iossa, Susanna

Iqbal, Tariq H.

Irini, Athanasaki

Irwin, Chris

Ishihara, Hisamitsu

Ishihara, Kengo

Ishii, Shinya

Iuliano, Sandra

Ives, Stephen

Iwahori, Toshiyuki

Iwai, Atsushi

Iwamori, Masao

Izawa, Kazuhiro P.

Izawa, Takeshi

Jaakkola, Johanna

Jaaro-Peled, Hanna

Jablonski, Heidrun 
Jackson, William F.

Jacobs, David

Jacobs, René

Jadeja, Ravirajsinh

Jager-Wittenaar, Harriet

Jahan-Mihan, Alireza

Jain, Ajay

Jakobsen, Jette

Jakubowicz, Daniela

Jalili, Thunder

James, Rob S.

Jamieson, Jennifer A.

Janda, Elzbieta

Jandacek, Ronald J.

Jang, Byoung Kuk

Jang, Hyeung-Jin

Janhonen, Kristiina

Jape, Gayatri

Jarvinen-Seppo, Kirsi

Jean-Marc Tadié, Jean-Marc

Jeckel, Kimberly M.

Jefferds, Maria Elena

JENA, PRASANT

Jenkins, David

Jensen, Megan E.

Jerebtsova, Marina

Jerković, Igor

Jessri, Mahsa

Jeżewska-Zychowicz, Marzena

Jiao, Li

Jin, Ran

Jirillo, Emilio

John, Katherine A.

Johnson, Claire

Johnson, Elizabeth

Johnson, Ian

Johnson, Stuart

Johnston, Carol

Jones, Julie

Jones, Kerry

Jones, Peter J. H.

Joo, Nam-seok

Jooken, Etienne

Joosten, Koen F.

Jordan, Jennifer

Jose, Pedro A.

Joven, Jorge

Jówko, Ewa

Joy, Jordan M.

Juan, Wenyen

Judd, Suzanne

Juge, Nathalie
Jukarainen, Sakari

Julia, Chantal

Julve, Josep

Juonala, Markus

Jurewitsch, Brian

Jurikova, Tunde

Juskiewicz, Jerzy

Juszczak, Lesław

Jyväkorpi, Satu K.

Kabil, Omer

Kagiya, Tadayoshi

Kahal, Hassan

Kahleova, Hana

Kaido, Toshimi

Kalanetra, Karen

Kalhan, Satish

Källén, Bengt

Kalliopi, Kotsa

Kalogirou, Charis

Kaludercic, Nina

Kamada, Nobuhiko

Kamat, Pradip

Kaminskyy, Vitaliy O.

Kamolz, Lars

Kanai, Yoshikatsu

Kancherla, Vijaya

Kandar, Roman

Kang, Jae Seung

Kantarci, Alpdogan

Kao, Erl-Shyh

Kapsokefalou, Maria

Karachaliou, Marianna

Karakochuk, Crystal

Karamichos, Dimitrios

Karatzi, Kalliopi

Karki, Rajendra

Karlsson, Erik

Karras, Spiros

Kasarda, Donald

Kašparovský, Tomáš

Kass, Lindsy S.

Katayama, Shigeru

Kato, Hiroyuki

Kato, Hisanori

Kato, Norihisa

Kattelmann, Kendra

Katuchova, Jana

Katz, Abigail

Kaur, Gunveen

Kawabata, Kyuichi

Kawai, Yoshichika

Kawano, Kouichiro 
Kayman, Kalantar-Zadeh

Kazankov, Konstantin

Kazlauskas, Andrius

Keating, Elisa

Keen, Douglas A.

Keenan, Michael

Keiler, Annekathrin Martina

Keller, Amélie

Keller, Markus

Kelley, Darshan

Kello, Martin

Kellow, Nicole J.

Kelly, Alan

Kennel, Julie

Kent, Katherine

Keshteli, Ammar Hassanzadeh

Kessler, Sonja

Khalesi, Saman

Khare, Sharad

Kharrazian, Datis

Khosla, Pramod

Khuder, Sadik A.

Kieliszek, Marek

Kiely, Mairead

Kienesberger, Petra

Kim, Chang $\mathrm{H}$.

Kim, Donguk

Kim, Hyounju

Kim, Jaehan

Kim, Jae-woo

Kim, Jung-Ae

Kim, Ju-Young

Kim, Kil-Soo

Kim, Mee Ree

Kim, Na-Hyung

Kim, Ok-Su

Kim, Sung-Hoon

Kim, Weon

Kimura, Tomoki

Kindler, Joseph

King, Janet

Kinsky, Suzanne

Kipp, Anna

Kippler, Maria

Kirk, Ben

Kirkpatrick, Sharon

Kishida, Taro

Kishimoto, Yoshimi

Kitic, Cecilia Mary

Kitissou, Marcel

Klatzkin, Rebecca R.

Klaus, Susanne
Kleber, Marcus E.

Kling, Pamela J.

Kłoda, Karolina

Kluger, Michael D.

Knapp, Caprice A.

Kneepkens, C. M. Frank

Knight, Alissa

Knipping, Karen

Knoell, Daren L.

Knol, Jan

Knott, Rachel

Knudsen, Knud Erik Bach

Kobayashi, Hanako

Koch, Manja

Koehler, Karsten

Koenigstorfer, Joerg

Koh, Gar Yee

Kojima-Yuasa, Akiko

Kok, Dieuwertje

Kok, Wouter

Kolahdooz, Fariba

Kolb, Andreas

Koleva, Petya T.

Kones, Richard

Kontny, Udo

Koon, Hon Wai

Koot, Bart G. P.

Kopjar, Mirela

Koplin, Jennifer

Koppe, Janna G.

Kornprat, Peter

Korosi, Aniko

Kotchen, Theodore A.

Kouassivi Aglago, Elom

Kouretas, Dimitrios

Koutelidakis, Antonios

Koutoukidis, Dimitrios

Koutsos, Athanasios

Kovatcheva-Datchary, Petia

Koyama, Yu

Krähenbühl, Stephan

Krau, Jeffrey A.

Krause, Rolfdieter

Krebs, Nancy

Kreider, Richard

Krejpcio, Zbigniew

Krogfelt, Karin

Kruger, Marlena

Krupa-Kozak, Urszula

Krupp, Danika

$\mathrm{Ku}$, Seockmo

Kuang, Zhizhou 
Kubena, Karen

Kubicova, Lenka

Kubis, Hans-Peter

Kubota, Naoto

Kuhlenschmidt, Mark

Kuk, Jennifer L.

Kukreja, Subhash

Kuligowski, Maciej

Kulozik, Ulrich

Kumar, Jothi Dinesh

Kuno, Toshiya

Kuo, Jen-Min

Kuo, Shiu-Ming

Kuo, Tony

Kurasaki, Masaaki

Kurrpa, Kalle

Kurz, Tino

Kusuda, Satoshi

Kuwabara, Masanari

Kuwahara, Atsukazu

Kuwahara, Keisuke

Kuwana, Tomomi

Kwak, Chung Shil

Kweon, Oh-Kyeong

Kwiatkowska, Katarzyna

Kwon, Oran

Kyro, Cecilie

Kyrozis, Andreas

La Favor, Justin D.

La Frano, Michael R.

La Vieille, Sébastien

Labruna, Giuseppe

Lac, Andrew

Lachenmeier, Dirk

Lacy, Katie

Lagouri, Vasiliki

Laguna, Joan-Carles

Laguzzi, Federica

Lahiri, Amit

Lai, I-Ju

Lai, Jun S.

Laird, Eamon

Laitinen, Kirsi

Lalas, Stavros

Lam, Yan Y.

Lamacchia, Carmela

Lammi, Carmen

Lamousé-Smith, Esi S. N.

Lamuela-Raventós, Rosa

Land, Mary-Anne

Landberg, Rikard

Landete, José Maria
Landis-Piwowar, Kristin

Landrier, Jean-françois

Lane, Darius

Langouche, Lies

Langsetmo, Lisa A.

Lanou, Amy Joy

Laparra, Jose Moisés

Larsen, Bodil M. K.

Lasekan, John B.

Lass, Achim

Latella, Giovanni

Laughlin, Maren

Lauritzen, Lotte

Lavender, Andrew

Laverty, Anthony A.

Lavoie, Jean-claude

Lawen, Alfons

Lawrence, Catherine B.

Lawrence, Karl

Layman, Donald

Le Couteur, David G.

Lê, Kim-Anne

Leach, Steven T.

Lecerf, Jean-michel

Lecoultre, Virgile

Ledda, Caterina

Lee, Hyeon Yong

Lee, Jason

Lee, Jun Hee

Lee, Junsoo

Lee, Karen Chia-Lun

Lee, Kwang Ho

Lee, Min Won

Lee, Ming-Fen

Lee, Ok-Hwan

Lee, Sang Gil

Lee, Sang-Han

Lee, Seung-Yeon

Lee, Sun Eun

Lee, Sun Young

Lee, Sun-Ok

Lee, Tzong-Shyuan

Lee, Vincent Ws

Lee, Yeon-Ju

Lefevre, Michael

Leffler, Jonatan

Legeza, Balázs

Lehtisalo, Jenni

Leite, João Costa

Lemieux, Simone

Lenzen, Sigurd

Leonard, Maureen M. 
Lephart, Edwin D.

Leri, Francesco

Lessard, Laura

Leung, Chung-Hang

Leung, Ricky Yuet-Kin

Levenson, Anait

Levinger, Pazit

Levinson, Ralph D.

Levy, Louis

Lewinska, Anna

Lewis, Nathan A.

Lewis, Rohan

Leyse-Wallace, Ruth

Li, Chun

Li, Duo

Li, Jau-Yi

$\mathrm{Li}, \mathrm{Li}$

Li, Qianjin

Li, Weijuan

Li, Wen-Wu

Li, Yan

Liao, Jyh-fei

Liao, Yi-Jen

Liaset, Bjørn

Liberato, Selma C.

Lidon, Fernando

Lieben, Cindy

Liem, Gie

Liguori, Maria

Lilenfeld, Lisa

Lim, Junxian

Lim, Karen

Lin, Chih-Chien

Lin, Chih-Li

Lin, Daniel

Lin, Hui-Hsuan

Lin, Pao-Hwa

Lin, Pei-Hui

Lin, Ping-Ting

Lin, Shih-Hua

Lin, Shyh-Hsiang

Lionetti, Vincenzo

Liou, Ying-ming

Lira, Fabio

Little, Tanya J.

Liu, Jian

Liu, Pei-Yang

Liu, Shing-Hwa

Liu, Yang

Livesey, Geoffrey

Livolti, Giovanni

Lo Monte, Attilio Ignazio
Lo Scalzo, Roberto

Locatelli, Francesco

Locatelli, Marcello

Löfblom, John

Loiselle, Denis S.

Løken, Elin B.

Lombardi, Angela

Lombardo, Caterina

Lonardo, Amedeo

London, Edra

Loor, Juan J.

Lopez Jornet, Pia

López, Luis Carlos

Lopez, Nanette

Lopez-Escamez, Jose A.

Lopez-Miranda, Jose

López-Sobaler, Ana M.

Lopez-Valcarcel, Beatriz Gonzalez

Lorenzo, Óscar

Lövestam, Elin

Lovreglio, Piero

Low, Dorrain

Lowe, Nicola

Lu, Louise Weiwei

Lubahn, Dennis

Lucey, Alice

Ludy, Mary-Jon

Lue, Ko-Huang

Luick, Bret R.

Lukaszuk, Judith

Lund, Elizabeth $\mathrm{K}$.

Lund, Jenny

Lundberg, Jon

Lundin, Knut

Lung, Ming-Yeou

Luo, Jin

Lupiáñez, José Antonio

Lutfi Royo, Esmail

Lutjohann, Dieter

Lutsey, Pam

Lutz, Thomas

Luzza, Francesco

Ly, Sun Yung

Lykkesfeldt, Jens

Lynch, Heidi M.

Lyons, Paul E.

Lysne, Vegard

M.Fuller, Patrick

Ma, David

Ma, Wenxin

Ma, Yunsheng

Ma, Zheng Feei 
MacFarlane, Amanda J.

Machetti, Fabrizio

Macia, Laurence

Mack, Isabelle

MacPherson, Rebecca

Madeddu, Roberto

Maeda, Akiko

Maehre, Hanne K.

Maestro, Miguel

Maffeis, Claudio

Mafra, Denise

Mager, Diana

Maggi, Davide

Maggini, Silvia

Mahajna, Jamal

Maher, Pamela

Mahimainathan, Lenin

Mahoney, Sara E.

Mai, Volker

Maiorino, Maria Ida

Maki, Kevin

Malavolta, Marco

Malemud, Charles J.

Malik, Rayaz A.

Malin, Steven K.

Mallard, Simonette R.

Mallo, Federico

Mandò, Chiara

Manganaris, George

Maniam, Jayanthi

Manni, Andrea

Manninger, Martin

Manoli, Irini

Mansueto, Pasquale

Manthou, Eirini

Mantzioris, Evangeline

Manuelli, Matteo

Marakis, Georgios

Marangoni, Franca

Marcadenti, Aline

Marchesini, Giulio

Marchini, Cristina

Maret, Wolfgang

Margaritelis, Nikos

Margerison, Claire

María, Tabernero Urbieta

Marigliano, Marco

Marín, Mercedes

Mariño, Eliana

Marino, Joseph

Mariotti, François

Markaki, Anastasia
Markey, Oonagh

Markhus, Maria Wik

Markou, Kostas. B.

Marks, Joanne

Marlicz, Wojciech

Marlow, Gareth

Marr, Robert

Marra, Maurizio

Marrs, Tom

Marshall, Skye

Marshall, Teresa A.

Marteau, Philippe

Martel, Fátima

Martin, Camilia

Martin, Finian

Martin, François-Pierre J.

Martín, Franz

Martin, Kristy

Martín-Antonio, Beatriz

Martinelli, Pasquale

Martínez, Homero

Martinez, Jessica

Martinez, Kristina

Martínez, Rosario

Martinez, Vicente

Martinez-Castelao, Alberto

Martini, Claudia

Martini, Daniela

Martín-Orúe, Susana M.

Martins, Ian James

Martins, Natália

Martorana, Alessandro

Maruyama, Kei

Marventano, Stefano

Marverti, Gaetano

Marx, Wolfgang

Marzullo, Paolo

Mascherini, Gabriele

Masilamani, Madhan

Maslin, Kate

Maslova, Ekaterina

Massot, Malen

Mastrocola, Raffaella

Masuelli, Laura

Matarese, Laura E.

Mateu De Antonio, Javier

Matheson, Jesse

Mathews, Suresh

Matias, Catarina

Matsubara, Keiichi

Matsumoro, Kenji

Matsumoto, Hiroyuki 
Matsuura, Bunzo

Matsuzaki, Keiichi

Matthys, Christophe

Mattijssen, Frits

Mattioli, Anna Vittoria

Mattner, Jochen

Maughan, Ronald J.

Maukonen, Mirkka

Mauriz, José L.

Mavian, Carla

Mavrogonatou, Eleni

May, Felicity

Mazoit, Jean-Xavier

Mc Naughton, Lars R.

McCaffrey, Tracy

McCarthy, Sinéad N.

McCaskill, Michael

McClain, Craig J.

McConnell, Glenn

McCourt, Peter

McDougall, Gordon

McGill, Anne-Thea

McKenna, Malachi J.

McLean, Rachael M.

McMahon, Emma

McMahon, Nick

Mcmillan, Donald C.

Mcmorrow, Tara

McNally, Dayre

McNeil, David

Meacham, Susan L.

Medler, Kathryn F.

Meilhac, Olivier

Mejía-Benítez, Aurora

Melby, Chris

Mellor, Duane D.

Mellott, Tiffany

Menaa, Farid

Mendes, Rosa

Méndez, Lucía

Méndez-Álvarez, Estefanía

Mendonça, Nuno

Meng, Hongdao

Meng, Yuan Xiang

Menkhorst, Ellen

Mercadel, Lucile

Mercado, Carla I.

Merchant, Anwar

Merkiel-Pawłowska, Sylwia

Mero, Antti

Merritt, Matthew

Messa, Pier Giorgio
Messaritakis, Ippokratis

Messier, Claude

Mettler, Samuel

Meyer, Barbara

Meyer, Katie A.

Meynier, Anne

Meyre, David

Mian, Caterina

Micek, Agnieszka

Michaelidou, Alexandra-Maria

Michalkiewicz, Martha

Michels, Alexander J.

Michigami, Toshimi

Micol, Vicente

Midgley, Adam

Miele, Luca

Mielenz, Manfred

Mielgo Ayuso, Juan

Mietlicki-Baase, Elizabeth G.

Migliaccio, Silvia

Mikhailov, Theresa A.

Miki, Yasuhiro

Milan, Amber

Milkovska-Stamenova, Sanja

Millen, Amy E.

Miller, Dr. Michael

Miller, Jacqueline

Miller, Mark

Mills, Kerry E.

Minami, Akira

Minato, Ken-ichiro

Minder, Elisabeth I.

Mineo, Chieko

Minicucci, Marcos F.

Minisola, Salvatore

Minjares-Fuentes, Jose Rafael

Mioso, Roberto

Miotto, Paula

Miralles, Beatriz

Miranda, Jonatan

Miranda, Jose M.

Mirmiran, Parvin

Misciagna, Giovanni

Mistretta, Antonio

Mistura, Lorenza

Miura, Kyoko

Miyata, Yasuyoshi

Miyazaki, Teruo

Miyazaki, Tetsuro

Miyazaki, Toru

Miyoshi, Eiji

Mizumura, Kazue 
Mizuno, Katsumi

Mizuno, Masashi

Mladěnka, Přemysl

Mlakar, Vid

Mocanu, Veronica

Moestrup, Søren Kragh

Mohajeri, M. Hasan

Mohamed, Suhaila

Molin, Marianne

Mollen, Kevin P.

Mond, Jon

Monda, Vincenzo

Monti, Daria Maria

Montilla, Antonia

Montoya, Carlos A.

Moon, Yuseok

Moor, Molly A.

Morabito, Antonino

Morabito, Rossana

Morales Suárez-Varela, María M.

Morán, José M.

Morán, Lara

Moranta, David

Moreau, Regis

Morelli, Lorenzo

Moreno, Diego A.

Moretti, Diego

Morganti, Pierfrancesco

Mori, Kiyoshi

Mori, Naoki

Morioka, Tomoaki

Morisset, Anne-Sophie

Morris, Claudia R.

Morris, Melanie

Morse, Nancy L.

Mosawy, Sapha

Moser, Othmar

Mosoni, Laurent

Most, Jasper

Moughan, Paul J.

Mougiakakou, Stavroula

Moukarzel, Sara

Moulder, John E.

Moulis, Jean-Marc

Mounier, Catherine

Mountjoy, Margo

Muhsen, Khitam

Mukhopadhyay, Partha

Mulhern, Maria S.

Mulinacci, Nadia

Müller, Manfred James

Müller, Thomas
Mulligan, Angela A.

Mulvihill, Erin

Munblit, Daniel

Muñiz, Pilar

Muñoz-Alférez, M. José

Murach, Kevin

Murgia, Chiara

Muriel, Pablo

Murotomi, Kazutoshi

Mursu, Jaakko

Murthi, Padma

Muscaritoli, Maurizio

Musumeci, Giuseppe

Muszyńska, Bożena

Mwinyi, Jessica

Myneni, Ajay

Myte, Robin

$\mathrm{Na}$, Huimin

$\mathrm{Na}$, Hye-Kyung

Nagai, Katshito

Nagai, Yoshinori

Nagao, Kenji

Nagaraj, Chandran

Nagasawa, Herbert

Nagata, Satoru

Nair-Shalliker, Visalini

Naito, Yuji

Nakahama, Ken-ichi

Nakajima, Atsushi

Nakayama, Masaru

Nakhauka, Ekesa Beatrice

Namork, Ellen

Naninck, Eva F. G.

Nanno, Masanobu

Naruishi, Koji

Nasser, Jennifer

Natarajan, Sathish Kumar

Naumovski, Nenad

Navarrete-Muñoz, Eva Maria

Navarro, Estanis

Navarro, Virginia

Navarro-Alarcon, Miguel

Navas-Carretero, Santiago

Naviglio, Silvio

Neacsu, Madalina

Neale, Elizabeth

Neale, Rachel E.

Neele, Dellschaft

Neelis, Esther G.

Neely, Greg

Negro, Massimo

Neilson, Andrew 
Nelson, Gregg

Nenna, Raffaella

Nestel, Paul

Neto, António W. Gomes

Neupane, Sudan Prasad

Newby, Ruth

Newnham, Evan

Nguyen, Tanya $\mathrm{T}$.

Ni Mhurchu, Cliona

Nicklas, Teresa

Nie, Peng

Nielsen, Forrest

Nielsen, Ole Haagen

Nieman, David C.

Niemeier, Andreas

Nieto, Gema

Nieves, Jeri W.

Nikawa, Takeshi

Niles, Richard M.

Nilsson, Ake

Nishi, Stephanie K.

Nissensohn, Mariela

Nistala, Ravi

Nobles, James

Nogués, M. Rosa

Nohr, Donatus

Nolden, Alissa

Nomikos, Tzortzis

Nomura, Yoshihiro

Nonn, Larissa

Nonnemacher, Michael R.

Nordgren, Tara

Norsa, Lorenzo

Norval, Mary

Noubissi, Felicite

Novellino, Ettore

Nowak, Felicia V.

Nowicka, Grażyna

Nowson, Caryl

Ntalli, Nikoletta G.

Nualart, Francisco

Nugent, Anne

Numazawa, Satoshi

Nybacka, Sanna

O'Hallora, Siobhan A.

O'Mahony, Liam

O'Malley, Dervla

Oaks, Brietta M.

Obana, Akira

O'Brien, Sarah H.

O'Connor, Eibhlís

Odland, Jon Øyvind
O'Gallagher, Kevin

Ogawa, Yukiharu

Oh, Sang-Ho

Ohlemiller, Kevin K.

Ohlsson, Bodil

Ohnishi, Masatoshi

Öhrvik, Veronica

Ohta, Takeshi

Ojala, Johanna O.

Ojalvo, Daniel Lozano

Ojima, Koichi

Oka, Shuntaro

O'kane, Gabrielle

O'Keefe, Stephen JD

Okuda, Masayuki

Okuda, Nagako

Olde Rikkert, Marcel G. M.

Oldewage-Theron, Wilna

Olendzki, Barbara

Oliveira, Karliana

Oliveira, Paula Alexandra Martins De

Oliveras-López, María-Jesús

Oliviero, Teresa

Olmedilla-Alonso, Begoña

Olney, Deanna K.

Olsen, Sjurður

Olza, Josune

Omagari, Katsuhisa

Omar, Syed Haris

Omotayo, Moshood

Oms-Oliu, Gemma

Onyenwoke, Rob U.

Opperhuizen, Anneloes

Orenes-Piñero, Esteban

Ormsbee, Michael

Ortega, Francisco José

Ortiz, Alberto

Osborne, Brenna

Oskarsson, Viktor

Östenson, Claes-göran

Ota, Tsuguhiro

O'Tierney-Ginn, Perrie

Otsuki, Takemi

Ou, Bor-rung

Oude Griep, Linda M.

Ouguerram, Khadija

Overvad, Thure Filskov

Ozawa, Mio

Padrão, Patrícia

Pagonopoulou, Olga

Paik, Jisun

Painter, Jodie N. 
Pal, Abhijeet

Pala, Valeria

Paller, Channing

Palmer, Amanda C.

Pammi, Mohan

Pan, Meixia

Pan, Min-Hsiung

Panagiotidis, Mihalis

Panaro, Maria Antonietta

Panza, Francesco

Panzella, Lucia

Paoli, Antonio

Paolo, Arosio

Papadakis, Sophia

Papadimitriou, Anastasios

Papadimitriou, Lampros

Papadimitriuou, Konstantinos

Papanikolaou, Yanni

Papetti, Adele

Parakhonskiy, Bogdan

Parhofer, Klaus G.

Park, Junsoo

Park, Yong Seek

Park, Yongsoon

Parkar, Shanthi G.

Parker, Helen

Parker, Leslie A.

Parlesak, Alexandr

Parnell, Jill

Parodi, Emilia

Parola, Maurizio

Parolini, Cinzia

Parr, Evelyn

Pasco, David S.

Pascual-Teresa, Sonia

Pasinetti, Giulio

Pasqui, Francesca

Passamonti, Sabina

Passarella, Daniele

Passaro, Angelina

Patel, Yashomati M.

Patinen, Pertti

Patole, Sanjay

Paton, Chad

Pazdro, Robert

Pearce, Elizabeth

Peart, Daniel J.

Pedraza-Chaverri, José

Pedrera-Zamorano, Juan D.

Pedret, Anna

Peery, Anne F.

Peiris, Madusha
Pellegrino, Daniela

Pelly, Fiona

Peluso, Ilaria

Peña Quintana, Luis

Peña, Amado Salvador

Peña, Maria Marjorette O.

Penagini, Francesca

Pena-Rosas, Juan Pablo

Penas, Sonia Eiras

Pence, Brandt

Peng, Chiung-Huei

Peng, Lifeng

Peng, Wen-Huang

Pentieva, Kristina

Penugonda, Kavitha

Pepe, Salvatore

Pepino, Marta Yanina

Peppelenbosch, Maikel

Percival, Susan

Pereira, David

Pereira, Dora

Pereira, Leonel

Pereira-da-Silva, Luís

Perez, Antonio

Perez, Jara

Perez-Cano, Francisco

Perez-Cornago, Aurora

Perez-Stable, Carlos

Periago, María Jesús

Perry, Tracy

Peters, John C.

Peters, Rachel L.

Peters, Simone

Petersen, Kristina S.

Peterson, Catherine A.

Peterson, Julia

Petroff, Brian K.

Pettersen, Caroline

Pettinato, Giuseppe

Peyer, Karissa

Pezzotto, Stella M.

Phang, Melinda

Philips, Neena

Phillips, Mary E.

Piccoli, Giorgina Barbara

Piccolo, Brian

Piche, Marie-Eve

Picklo, Matthew J.

Piemontese, Luca

Pierce, Grant

Pietrella, Donatella

Pietrobelli, Angelo 
Pignitter, Marc

Pignotti, Giselle

Piknova, Barbora

Pilon, Marc

Pilz, Stefan

Pinent, Montserrat

Pirola, Luciano

Piso, Rein Jan

Piva, Terrence

Pivovarova, Olga

Plaza-Díaz, Julio

Plazas Ávila, Maria De La O

Plochocki, Jeffrey H.

Plösch, Torsten

Polanska, Kinga

Polidori, Maria Cristina

Pollock, Richard L.

Poole, David C.

Popovich, David

Poquet, Laure

Porres, Jesús M.

Portengen, Lützen

Porter, Judi A.

Posovszky, Carsten

Potenza, Lucia

Poti, Jennifer

Potischman, Nancy

Potter, Julia

Potthoff, Matthew J.

Poudyal, Hemant

Pourshahidi, Kirsty

Power, Madeleine

Powers, Jacquelyn M.

Powers, James S.

Powles, John

Pozzilli, Paolo

Pravst, Igor

Preitner, Frederic

Premkumar, Muralidhar

Pribus, Peter

Prieto-Lloret, Jesus

Primeaux, Stefany D.

Probert, Chris

Proestos, Charalampos

Pugliese, Giuseppe

Purchas, Roger W.

Puri, Basant K.

Pursey, Kirrilly

Purwaha, Preeti

Püschel, Gerhard P.

Qasem, Wafaa A.

Qin, Bo
Quadros, Edward V.

Quigley, Eamon

Quintero-Florez, Angelica

Raatz, Susan K.

Rachaputi, Rao C. N.

Rachek, Lyudmila

Rachoń, Dominik

Radmacher, Paula

Radyukina, N. L.

Raimann, Jochen G.

Rainger, George Edward

Rajakumar, Kumaravel

Raju, Raghavan

Rallabhandi, Prasad

Raman, Jay

Ramdath, Dan

Ramos Campo, Domingo Jesús

Ramos, Sonia

Rana, Brinda K.

Ranganathan, Natarajan

Ranjit, Nalini

Ranzato, Elia

Rao, Shripada

Rapoport, Stanley I.

Rathinavelu, Appu

Rattan, Suresh

Ravallec, Rozenn

Ray, Seemun

Raz, Olga

Reaney, Martin J. T.

Rees, Bill

Reeve, Belinda

Reeve, Vivienne

Rege, Shraddha

Reger, Michael

Rehm, Colin

Reicks, Marla

Reidlinger, Dianne

Reimer, Raylene

Reinblatt, Shauna P.

Reiner, Martin

Reis, Flávio

Reiser, Jochen

Reisman, Scott A.

Reitelseder, Søren

Reiter, E. Miranda

Remacha, Angel F.

Ren, Jianguo

Renaud, Justine

Renner, Wilfried

Repa, Andreas

Resch, Bernhard 
Resiere, Dabor

Revuelta Iniesta, Raquel

Rey, Federico

Rezamand, Pedram

Rezzani, Rita

Rhodes, Jonathan

Ribas-Barba, Lourdes

Ricciardelli, Carmela

Richard, Caroline

Richardson, Alan

Riddlle, Lynn

Riedel, Bernhard

Riedel, Christian U.

Rienecke, Renee

Riesco, Eléonor

Rigo, Jacques

Rigottier-Gois, Lionel

Riis, Simon

Riley, Malcolm

Rimbach, Gerald

Ringel-Kulka, Tamar

Rink, Lothar

Rios-Covian, David

Riscuta, Gabriela

Rivellese, Angela A.

Rivero-Pérez, M. Dolores

Rizzarelli, Enrico

Rizzo, Angela Maria

Robberecht, Harry

Robert-Da Silva, Christine

Roberts, Rosebud O.

Robertson, Denise

Robertson, Jeremy

Robertson, Kirsten

Robitaille, Julie

Robles, Brenda

Robson, Shannon M.

Rodrigues, Isabel

Rodriguez Artalejo, Fernando

Rodríguez, Alexander J.

Rodríguez, María Correa

Rodríguez, Mariano

Rodriguez-Herrera, Alfonso

Rodriguez-Mateos, Ana

Roe, Brian

Roelofs, Erica

Roggero, Paola

Rohner, Fabian

Rohrmann, Sabine

Romanelli, Francesco

Romani, Andrea

Romeo, Stefano
Ronco, Pierre

Rondanelli, Mariangela

Root, Martin M.

Rosado, Catarina

Rose, Devin

Rosendahl, Jonas

Rosendale, Douglas Ian

Rosenlund, Helen

Rosenthal, Philip

Rosinger, Asher

Ross, Alastair

Rosset, Robin

Rossi, Filippo

Rossi, Mauro

Rossi, Paola

Rossiter, John

Rostaing, Lionel

Rostami, Kamran

Rother, Kristina

Rouhiainen, Ari

Rousseau, Guy

Rousselot, Bonnefont

Rowland, Ian

Roychowdhury, Sanjoy

Roza, Sabine J.

Rozen, Rima

Rozhon, Wilfried

Rubio-Arraez, Susana

Rucklidge, Julia

Ruddock, Helen

Rudkowska, Iwona

Rudolph, Bryan

Rudolph, Michael C.

Rudzitis-Auth, Jeannette

Rufian Henares, Josè Angel

Ruscica, Massimiliano

Rush, Elaine C

Russell, Alexandra C.

Russell, Fraser D.

Russell, Mark

Rutegård, Martin

Saarela, Maria

Sabate, Juan

Sacks, Gordon

Sadanaga, Tsuneaki

Saha, Shyamali

Sahay, Bikash

Saikumar, Pothana

Sainsbury, Kirby

Saisho, Yoshifumi

Sakhaee, Khashayar

Sala, Roberto 
Salaheen, Serajus

Salehi, Ahmad

Salim, Samina

Salis, Amanda

Salles, Christian

Salmerón, Ivan

Salomone, Federico

Salvatore, Francesco

Salvo, Andrea

Samala, Niharika R.

Samaniego Vaesken, María De Lourdes

Samuel, Chrishan

Samuelsson, Anne-Maj

San Martin, Carmen

Sanchez, Christelle

Sánchez-Fidalgo, Susana

Sanchez-Muniz, Francisco J.

Sandell, Mari

Sanders, Kerrie M.

Sanders, Tom

Sandvik, Pernilla

Sankavaram, Kavitha

Sanmartin, Chiara

Santé-Lhoutelliera, Veronique

Santoro, Luca

Santoro, Nicola

Santos, Debora

Santulli, Gaetano

Sanz-Paris, Alejandro

Sarris, Jerome

Sassi, Yassine

Sastre-Serra, Jorge

Sato, Daisuke

Sato, Kenji

Sato, Shin

Saturnino, Carmela

Sauder, Katherine Ann

Saunders, Bryan

Savary-Auzeloux, Isabelle

Savion, Naphtali

Saw, Constance L. L.

Sayón-Orea, Carmen

Scano, Paola

Scaramuzza, Andrea E.

Scarmeas, Nikolaos

Scazzina, Francesca

Schachter, Michael

Schag, Kathrin

Schauss, Alexander

Scheers, Nathalie M.

Scheffler, Christiane

Scherf, Katharina
Schiavone, Stefania

Schild, Lorenz

Schini-Kerth, Valérie

Schirmer, Bastian

Schlegel, Amnon

Schloss, Janet M.

Schmetterer, Leopold

Schmidt, Ricarda

Schnackenberg, Laura

Schoeler, Natasha E.

Schoeller, Dale

Schoenaker, Danielle A. J. M.

Schonthal, Axel

Schulz, Kalynn M.

Schulze, Kerry J.

Schupp, Michael

Schwab, Ursula

Schwartz, Gary G.

Schwetz, Verena

Scicchitano, Pietro

Scoffield, Jessica

Scott, Fraser W.

Scott, Sam

Scovassi, Anna Ivana

Scragg, Robert

Scudiero, Olga

Sculley, Dean V.

Seal, Judy

Seale, Lucia

Searle, Samuel D.

Sears, Barry

Sebastian, Rhonda

Sechi, Leonardo A.

Seddon, Johanna M.

Segovia-Siapco, Gina

Sekikawa, Akira

Sekulic, Damir

Sellayah, Dyan

Selmin, Ornella I.

Selvam, Chelliah

Sena, Cristina M.

Senchina, David

Serio, Francesco

Serra, Andrea

Serra, Dolors

Serra, Francisca

Serralheiro, Maria L.

Serra-Majem, Lluis

Serrano, Jose C. E.

Serrat, Maria A.

Sethi, Gautam

Setzer, William N. 
Seubert, John M.

Seyssel, Kevin

Sha, Zhe

Shackelford, Rodney E.

Shah, Dilip

Shah, Romina

Shah, Savit. H.

Shah, Zahoor

Shahar, Danit

Shaikh, Saame Raza

Shakibaei, Mehdi

Shanely, R. Andrew

Shankar, Eswar

Shankar, Padmini

Shao, Andrew

Shapses, Sue

Sharma, Bhesh

Sharma, Mukut

Sharma, Naveen

Sharma, Rohit

Sharma, Sushil

Sharma, Umakant

Sharp, Paul

Shecterle, Linda M.

Shen, Leslie

Shen, Qiang

Shen, Szu-Chuan

Sheridan, Paul O.

Shertzer, Howard G.

Shewale, Swapnil

Shi, Suan

Shiao, Young-Ji

Shida, Kan

Shidoji, Yoshihiro

Shimada, Yasuhito

Shimizu, Mitsuru

Shimizu, Takahiko

Shimosawa, Tatsuo

Shin, Andrew C.

Shin, Dayeon

Shipman, Kate

Shirin, Ziaei

Sholkamy, Eman

Shukla, Alpana P.

Siega-Riz, Anna Maria

Silswal, Neerupma

Silva, Branca M.

Silva, Robin

Silverberg, Donald S.

Silvestre, Marta Filipa Paulino

Sim, Kyra

Simko, Fedor
Simon, Marie-Christine

Simoni, Jan

Simopoulos, Artemis P.

Simpson, Melanie Rae

Singer, Pierre

Singh, Anil

Singh, Ashok K.

Singh, Ashutoush

Singh, Chandra K.

Singh, Nagendra

Singh, Udai P.

Singh, Vijay P.

Singh, Vishal

Singhal, Vibha

Siniscalco, Dario

Sirtori, Cesare

Sjöberg, Klas

Sjostrom, Elisabeth Stoltz

Skaaby, Tea

Skeaff, Sheila

Skelton, Matthew R.

Slater, Joyce

Sleigh, Alison

Słoczyńska, Karolina

Sluik, Diewertje

Sluimer, Judith

Smith, Caren

Smith, Claire

Smith, Gordon

Smith, Jill P.

Smith, Michael

Smith, Taryn

Smoliga, James

Smutzer, Gregory

Snaedal, Sunna

Snih, Soham Al

Soares, Mario

Socha, Katarzyna

Söderström, Lisa

Soenen, Stijn

Sohn, Uy Dong

Sokół-Łętowska, Anna

Solà Alberich, Rosa

Solah, Vicky A.

Solanas, Montserrat

Somoza, Beatriz

Song, Jaewhan

Song, Moon K.

Song, Sang-Wook

Song, YoonJu

Sonoyama, Kei

Sopade, Peter A. 
Soraisham, Amuchou S.

Soreq, Hermona

Sornay-Rendu, Elisabeth

Sotomayor, Camilo G.

Sotos-Prieto, Mercedes

Soueidan, Assem

Soulage, Christophe

Sourij, Harald

Souza-Smith, Flavia

Soybel, David I.

Spegazzini, Nicolas

Spégel, Peter

Spence, J. David

Spiro, Ayela

Spitznagel, Mary Beth

Spriet, Lawrence

Stacchiotti, Alessandra

Stadlbauer, Vanessa

Stadler, Diane D.

Stafford, Lorenzo D.

Stagos, Dimitrios

Stamm, Rosemary

Stanforth, Phil

Stangl, Gabriele

Stanley, Dragana

Stanley, Roger

Stannard, Stephen

Starek, Małgorzata

Starup-Linde, J.

Stauber, Rudolf E.

Staudacher, Heidi

Stautz, Kaidy

Stea, Tonje H.

Steenson, Simon

Stefanidou, Maria E.

Stefanon, Bruno

Stefler, Denes

Stegmayr, Bernd

Stehle, Peter

Steil, Garry M.

Stengel, Andreas

Stephen, Ian

Stevens, Paul E.

Stevens, Rebekah

Stewart, Maria

Steyn, Frederik

Stilli, Donatella

Stobdan, Tsering

Stokes, Caroline S.

Stomby, Andreas

Stork, Christian J.

Stough, Cathleen
Stove, Christophe P.

Strasser, Barbara

Stratakis, Nikos

Strazzullo, Pasquale

Strekalova, Tatyana

Strien, Tatjana Van

Stringham, James M.

Stuart, Sam

Stubbs, Brianna

Stuetz, Wolfgang

Stull, April

Stumbo, Phyllis J.

$\mathrm{Su}, \mathrm{Shu}-J e m$

$\mathrm{Su}$, Xiao Q.

Subar, Amy F.

Subhan, Fatheema Begum

Sui, Zhixian

Sukocheva, Olga

Sukumar, Deeptha

Sukumaran, Sunil Kumar

Suleria, Hafiz Ansar

Suliburska, Joanna

Sullivan, Margaret

Sun, Feng-Hua

Sun, Qian

Sung, Valerie

Suwa, Michihiro

Suzuki, Katsuhiko

Sweazea, Karen

Świeca, Michał

Sylvetsky, Allison

Szafron, Michael

Szászi, Katalin

Szepfalusi, Zsolt

Szewczyk, Katarzyna

Szilagyi, Andrew

Szkudelski, Tomasz

Szlagatys-Sidorkiewicz, Agnieszka

Szychlinska, Marta Anna

Taghibiglou, Changiz

Tahrani, Abd A.

Tain, You-Lin

Tajiri, Yuji

Takahama, Umeo

Takahashi, Satoshi

Takahisa, Kanekiyo

Takatani-Nakase, Tomoka

Takaya, Junji

Takayama, Fusako

Takechi, Ryusuke

Takeda, Eiji

Takimoto, Hidemi 
Tako, Elad

Talati, Zenobia

Tamura, Yukinori

Tang, Minghua

Tao-Hsin, Tung

Taoka, Yousuke

Tappia, Paramjit S.

Tappy, Luc

Tarantino, Giovanni

Tarocchi, Mirko

Tasevska, Natasha

Tashiro, Hirotaka

Tauler, Pedro

Taupin, Doug

Tavernarakis, Nektarios

Tawia, Susan

Taylor, Christopher A.

Taylor, Kathryn

Taylor, Rachael

Taylor, T. Matthew

Te Morenga, Lisa

Teas, Jane

Teasdale, Scott

Tecce, Mario Felice

Tekle, Michael

Telang, Sucheta

Temeyer, Kevin B.

Temme, Elisabeth H. M.

Temple, Norman

Teodorowicz, Małgorzata

Tepper, Beverly J.

Ter Horst, Kasper

Tersey, Sarah A.

Teske, Jennifer

Tesoriere, Giovanni

Tessier, Frederic J.

Teuber, Ramona

Tey, Siew Ling

Thangaratinam, Shakila

Then, Cornelia

Thèvenod, Frank

Thijs, Carel

Thomas, Diana

Thomas, Helen E.

Thomas, Lynn K.

Thompson, Henry

Thomsen, Hauke

Thomson, Jasmine

Thornley, Simon

Thornley-Brown, Denyse

Thunders, Michelle

Thurnham, David
Thyagarajan, Baskaran

Tielemans, Myrte J.

Tierney, Audrey

Timmerman, Kyle L.

Tinker, Sarah C.

Tintle, Nathan

Tipoe, George

Tipton, Kevin

Tissing, Wim

Titorenko, Vladimir I.

Titze, Jens Marc

Tiwari, Sanjay

Toldo, Stefano

Toledo, Alvaro

Toledo, Estefania

Toledo-Corral, Claudia

Tomasello, Giovanni

Tomaszewska, Ewa

Tong, Iris

Toorie, Anika

Torquati, Luciana

Torres López, Ma Isabel

Torres Perales, Carolina

Torres, Susan

Toshimitsu, Takayuki

Tosi, Paola

Toubekis, Argyris G.

Touil-Boukoffa, Chafia

Tournier, Carole

Tovar, Juscelino

Tovoli, Francesco

Townend, Jonathan

Traber, Maret

Traina, Giovanna

Tran, Cuong

Tran, Pamela

Tranchant, Carole

Traustadóttir, Tinna

Trehan, Indi

Tremblay, Angelo

Trenell, Michael

Tresserra-Rimbau, Anna

Trevena, Helen

Triaca, Viviana

Triantafyllou, Konstantinos

Trico, Domenico

Trieu, Kathy

Trif, Monica

Triunfo, Stefania

Troesch, Barbara

Troise, Antonio Dario

Trojanowicz, Bogusz 
Trombetta, Domenico

Trommelen, Jorn

Tronel, Claire

Trovato, Guglielmo

Trovik, Jone

Troy, Lisa

Truong, Khoa

Tsai, James

Tsao, Francis

Tschon, Matilde

Tselepis, Chris

Tseng, Ching-Jiunn

Tseng, Tung-Sung

Tsiani, Evangelia

Tsivgoulis, Georgios

Tsuzuki, Tsuyoshi

Tuck, Caroline

Tucker, Larry A.

Tucker, Robin

Tueros, Itziar

Tufarelli, Vincenzo

Tur, Josep Antoni

Turesky, Robert

Turiel, Maurizio

Turner, Justine

Turner, Raymond

Turner, Russell T.

Turpin-Nolan, Sarah

Tursi, Antonio

Tuuri, Georgianna

Tuvdendorj, Demidmaa

Tylavsky, Frances A.

Tyler, Churchward

Tyson, Crystal Cenell

Tyson, Darren R.

Tyunin, Alexey P.

Tzakos, Andreas G.

Tzanakis, Ioannis P.

Tziomalos, Konstantinos

Ubago-Guisado, Esther

Uberti, Daniela

Uccella, Nicola A.

Uchida, Yoshikazu

Uebanso, Takashi

Uehara, Mariko

Uehara, Tomoya

Uhal, Bruce D.

Ujcic-Voortman, Joanne

Ulatowski, Lynn

Ullrich, Reiner

Umano, Giuseppina Rosaria

Unno, Keiko
Upadhyaya, Jasbir

Urrechaga, Eloísa

Uusi-Rasi, Kirsti

Uusitupa, Matti

Vajro, Pietro

Valacchi, Giuseppe

Valentão, Patrícia

Valentini, Luzia

Valenzuela, Rodrigo

van Baak, Marleen

Van Ballegooijen, Adriana J.

Van Ballegooijen, Hanne

Van Den Engel-Hoek, Lenie

Van Den Heuvel, Ellen GHM

Van Der Haar, Frits

Van Der Kamp, Jan Willem

Van Etten, Jamie

Van Griensven, Leo

Van Guelpen, Bethany

Van Raay, Terry

Van Schothorst, Evert M.

Van Wijk, Nick

Van Zanten, Arthur R. H.

Vance, David E.

Vance, Terrence

Vandenboom, Rene

Vandenplas, Yvan

Vander Wal, Jillon

Vanderhoof, Jon A.

Vangelista, Luca

Varady, Krista

Varela-López, Alfonso

Varela-Moreiras, Gregorio

Vargas, Jorge $\mathrm{H}$.

Varraso, Raphaëlle

Vashist, Yogesh Kumar

Vásquez, Elizabeth

Vassalle, Cristina

Vassallo, Matteo

Vaughan, Roger A.

Vaughn, Mathew

Vecchione, Carmine

Veerman, J. Lennert

Veleeparambil, Manoj

Venancio, Vinicius Paula

Venema, Koen

Venkatadri, Rajkumar

Venkatesha, Shivaprasad

Venn, Bernard

Venter, Carina

Ventrella, Domenico

Venturi, Francesca 
Verain, Muriel C. D.

Verbeke, Kristen

Verd, Sergio

Vergara, Daniele

Verkaik-Kloosterman, Janneke

Vetter, Stefan W.

Vetvicka, Vaclav

Via, Michael A.

Vicente-Salar, Néstor

Vieira-Potter, Victoria

Viennois, Emilie

Vierucci, Francesco

Vignini, Arianna

Viljakainen, Heli T.

Villa-Bellosta, Ricardo

Villani, Anthony

Villano, Debora

Villarini, Anna

Villarreal, Jose Trevino

Vilstrup, Hendrik

Vincent, Grace

Vincent, John B.

Vinceti, Marco

Vinchi, Francesca

Vinciguerra, Manlio

Vinson, Joe

Vioque, Jesús

Virtanen, Jyrki

Visioli, Francesco

Visser, Anita

Vita, Roberto

Vitale, Marilena

Vitetta, Luis

Vitturi, Dario

Vlassopoulos, Antonis

Vogel, Christina

Volpato, Milene

Von Hundelshausen, Phillip

Von Hurst, Pamela

Von Lintig, Johannes

Von Schacky, Clemens

Von Wright, Atte

Voortman, Trudy

Vormann, Jürgen

Voznesenskaya, Anna

Wada, Jun

Wadehra, Madhuri

Wagner, Anika

Wahls, Terry

Wainford, Richard D.

Wakabayashi, Hidetaka

Waligora-Dupriet, Anne-Judith
Walker, Alan

Walker, Christie

Walker, D. Catherine

Walker, Marjorie. M.

Wallace, Taylor C.

Walsh, Jennifer M.

Walton, Gemma Emily

Walyaro, D. J.

Wanders, Desiree

Wang, Bo

Wang, Chi Chiu

Wang, Chin-Kun

Wang, Dong

Wang, Feng-Sheng

Wang, Guozheng

Wang, Liang

Wang, Xinkun

Wang, Yuming

Wang, Zhanwei

Ward, Leigh

Wardenaar, Floris C.

Wargovich, Michael

Warr, David G.

Wasse, Lucy

Waterlander, Wilma

Wätjen, Wim

Watkins, Adam J.

Watkins, Colleen Marie

Watkins, Julia

Watrowski, Rafał

Watson, William

Watt, Peter

Wax, Benjamin

Weaver, Connie

Weber, Janet

Weber, Lynn

Weghuber, Daniel

Wei, Gan

Weickert, Martin O.

Weiergräber, Marco

Weiler, Hope

Weimann, Arved

Weissgerber, Tracey L.

Welham, Simon

Wellard-Cole, Lyndal

Welsh, Jean A.

Welsh, Michael

Welters, Hannah J.

Wendin, Karin

Wendler, Christopher

Weng, Han-Rong

Wentz, Laurel M. 
Wertz, Philip W.

Wesson, Donald

West, Daniel

West, Keith

Westenbrink, Susanne

Westerberg, Ane Cecilie

Whisner, Corrie M.

White, John S.

White, Sarah H.

Whitfield, Patricia

Whiting, Susan

Whybrow, Stephen

Widmer, Hans Rudolf

Wierzejska, Regina

Wieser, Simon

Wightman, Emma L.

Wilkinson, Jenny M.

Willems, Mark

Williams, Andrew R.

Williams, Christopher S.

Williams, Elizabeth

Williams, Nancy

Williams, Robert J.

Williamson, Gary

Williamson, Kevin

Wilson, Patrick

Wilson, Ted

Winham, Donna

Winkler, Marion F.

Wirth, Michael

Wise, Paul

Witt-Enderby, Paula

Witting, Paul K.

Wittmann, Gábor

Woelwer-Rieck, Ursula

Wolber, Fran

Wolfe, Robert R.

Wölnerhanssen, Bettina Karin

Wolters, Maike

Won, Kyu Chang

Wondrak, Georg T.

Wong, Man-Sau

Wong, Rachel

Woo, Jessica Graus

Worsley, Anthony

Woźniak, Łukasz

Wright, Melecia

Wu, Chi-Rei

$\mathrm{Wu}$, Feitong

$\mathrm{Wu}, \mathrm{Li}-\mathrm{Chen}$

$\mathrm{Wu}, \mathrm{Tzu}-\mathrm{Hua}$

$\mathrm{Wu}$, Wen-Tzu
Wulf, Hans Christian

Wullaert, Andy

Wunderlich, Shahla M.

Wydau, Sandra

Xavier, Cristina Pinto Ribeiro

Xiaoli, Alus

Xie, Guoxiang

Xinyin, Jiang

Xiong, Zhaohui

$\mathrm{Xu}$, Feng-Lian

$X u$, Hang

$\mathrm{Xu}$, Jing

Xue, Xiang

Xun, Pengcheng

Yabe, Daisuke

Yamada, Yosuke

Yamaguchi, Yasuhiro

Yamaki, Kohji

Yamamoto, Takayuki

Yamanaka-Okumura, Hisami

Yamazaki, Etsuko

Yamazaki, Tomomi

Yan, Bowen

Yang, Gong

Yang, Hsin-Yi

Yang, Jae Wook

Yang, Qiyuan

Yang, Ray-Yu

Yang, Rong-Sen

Yang, Woong-Mo

Yang, Yue

Yannicelli, Steven

Yano, Shozo

Yaqoob, Parveen

Yarmolinsky, Ludmila

Yasuda, Kazuki

Yatabe, Junichi

Yatera, Kazuhiro

Yeates, Alison

Yee, Jennifer K.

Yeh, Kuo-Wei

Yeh, Shu-Lan

Yeh, Sung-Ling

Yi, Stella

Yian, Gu

Yiannakopoulou, Eugenia

Yim, Jung-Eun

Yimam, Mesfin

Yoder, Mervin

Yohei, Shirakami

Yokota, Tomoya

Yolton, Kimberly 
Yoneyama, Tadakatsu

Yoshida, Tadashi

Yoshihito, Yokoyama

Yoshinaga, Jun

Yoshino, Jun

Yoshiyama, Hironori

Young, Antony

Yu, Chia-Li

Yu, Siwang

$\mathrm{Yu}$, Zhiping

Yuan, Lijuan

Yui, Kunio

Zaborskis, Apolinaras

Zafeiridis, Andreas

Zakrzewski-Fruer, Julia

Zamora-Ros, Raul

Zand, Nazanin

Zang, Liqing

Zanini, Barbara

Zanon-Moreno, Vicente

Zanotti, Ilaria

Zarogoulidis, Paul

zastre, jason

Zavacki, Ann-Marie

Zavattari, Patrizia

Zdunczyk, Zenon

Zeinstra, Gertrude

Zeman, Daniel Meier

Zemel, Michael

Zeng, Huawei

Zevallos, Victor F.

Zgaga, Lina

Zhang, Bing
Zhang, Deqiang

Zhang, Hua

Zhang, Jian

Zhang, Jianjun

Zhang, Liping

Zhang, Yumei

Zhao, Liang

Zheng, Jolene

Zheng, $\mathrm{M}$.

Zheng, Wenxin

Zhou, Jingying

Zhu, Chris

Zhu, Fengqing Maggie

Zhu, Hua

Zhu, Zongjian

Zhuang, Shougang

Ziegler, Ekhard

Ziegler, Jane

Zilversmit, Leah

Zimmermann, Petra

Zinman, Bernard

Zittermann, Armin

Zivkovic, Angela

Zlatko, Nikoloski

Zmijewski, Michal

Zock, Peter L.

Zordi, Nicola De

Zosky, Graeme

Zou, Ping

Zuccotti, Gian V.

Zuhl, Micah

Zwilling, Chris

(C) 2018 by the authors. Licensee MDPI, Basel, Switzerland. This article is an open access article distributed under the terms and conditions of the Creative Commons Attribution (CC BY) license (http://creativecommons.org/licenses/by/4.0/). 